\title{
MHD simulation of the formation and propagation of multiple magnetic clouds in the heliosphere
}

\author{
Y. Wang, H. Zheng, S. Wang, and P. Ye \\ School of Earth \& Space Sci., Univ. of Sci. \& Tech. of China, Hefei, Anhui 230026, PR China \\ e-mail: ymwang@ustc.edu.cn \\ Received 6 June 2004 / Accepted 22 November 2004

\begin{abstract}
A multiple-magnetic-cloud (Multi-MC) structure formed by the overtaking of two successive coronal mass ejections (CMEs) in the heliosphere is studied by using a 2.5-D MHD simulation. This simulation illustrates the process of the formation and propagation of two identical CMEs, which are ejected with speeds of $400 \mathrm{~km} \mathrm{~s}^{-1}$ and $600 \mathrm{~km} \mathrm{~s}^{-1}$ respectively and initially separated by $12 \mathrm{~h}$. The results show that it takes $\sim 18 \mathrm{~h}$ for the fast cloud to catch up with the preceding slow one, then the two clouds form a Multi-MC structure that arrives at $1 \mathrm{AU}$ three days later. The fast cloud is slowed down significantly because of the blocking by the preceding slow one. This implies that the travel time of a Multi-MC structure is dominated by the preceding slow cloud. Moreover, most primary observational characteristics of Multi-MC at $1 \mathrm{AU}$ are well represented by the simulation. In addition, by combining observations, theoretical model and the simulation results, differences between Multi-MC and other types of in-situ observed double-flux-rope structure are addressed. A comparison of Multi-MC to coronalmass-ejection cannibalization near Sun is also given.
\end{abstract}

Key words. magnetohydrodynamics (MHD) - methods: numerical - Sun: coronal mass ejections (CMEs) interplanetary medium - standards

\section{Introduction}

Magnetic clouds (MCs) as a consequence of coronal mass ejections (CMEs) play an important role in solar-terrestrial physics. Through in-situ spacecraft measurements, a magnetic cloud manifests three definite characteristics (e.g., Burlaga et al. 1981): enhanced magnetic field strength, smooth rotation of the field vector and a low ratio of proton thermal pressure to magnetic pressure, $\beta$. A magnetic cloud expands when it propagates in the interplanetary medium. Statistically, its expansion speed is $\sim 1 / 2$ of the local Alfvén speed (Klein \& Burlaga 1982) and the diameter of a typical cloud, at $1 \mathrm{AU}$, is $\sim 0.28 \mathrm{AU}$ (Lepping et al. 1990).

It is suggested that magnetic clouds can be described by the flux rope model, in which a loop-like cloud is still connected with the solar surface (Larson et al. 1997). In many situations, a magnetic cloud is ideally depicted as a local cylinder with a constant $\alpha$ force-free field (e.g., Goldstein 1983; Burlaga 1988; Farrugia et al. 1993; Kumar \& Rust 1996; Osherovich \& Burlaga 1997). Based on the local cylindrical flux rope model, some numerical simulations have been carried out, where isolated loop-like magnetic clouds with various magnetic field strengths, axis orientations and speeds have been investigated (e.g., Vandas et al. 1995, 1996, 1997). A great consistency was found between the in-situ observations, theoretical analyses and simulations.
In addition to the isolated ejecta, complex ejecta formed by the multiple CMEs overtaking each other (e.g., Burlaga et al. 1987, 2001) are often observed in the solar wind. Multiple ejections may originate from the same solar source (Gopalswamy et al. 2002a; Gopalswamy 2003) or from different solar source regions (Gopalswamy et al. 2002b). For an isolated magnetic cloud, the configuration is simple because it only interacts with the ambient solar wind, therefore it propagates and expands relatively freely. But for complex ejecta the situation becomes more complicated because of the interactions between multiple ejections. Such interactions result in a new class of nonthermal radio emission, and make a possible contribution to generating solar energetic particle events (Gopalswamy et al. 2001, 2002a,b).

Since most complex ejecta contain disordered magnetic fields, no theoretical model can be effectively used to describe them except the multiple-magnetic-cloud (Multi-MC) structure, a particular kind of complex ejecta. A Multi-MC, proposed and proved by Wang et al. $(2002,2003)$, is formed by a series of successive MCs. Different from other complex ejecta, a Multi-MC comprises several sub-clouds and interacting regions between sub-clouds. According to the observations of three cases of Multi-MC during March-April 2001, each subcloud within Multi-MC primarily satisfies the criteria of a typical magnetic cloud. In the interacting region, the field strength decreases to minimum, and the proton temperature and $\beta$ 
increase simultaneously. Hence, a Multi-MC contains a regular magnetic field. Although a Multi-MC consists of several clouds, its scale approaches that of a typical isolated magnetic cloud. The above observational results imply that a Multi-MC is compressed heavily and is potentially extremely geoeffective (Wang et al. 2004a).

Since the sub-clouds inside a Multi-MC are relatively independent, Wang et al. (2002, 2003) applied a combination of several flux ropes to model Multi-MCs. The result of the fit is consistent with the in-situ observations at $1 \mathrm{AU}$. It is obvious that the magnetic field configuration of a Multi-MC varies with different combinations of sub-clouds, and the impacted combination factors include the sign of the sub-cloud helicity, the orientation of the sub-cloud axis, the number of sub-clouds, and so on. A Multi-MC is a relatively new concept that has not been studied comprehensively. Wang et al. (2002) tried to theoretically analyze the characteristics of double $\mathrm{MC}$, which is the simplest case of a Multi-MC, but could not give a picture of its formation and propagation in the heliosphere. In this paper, we will study it using a 2.5 dimensional MHD simulation.

When a spacecraft goes through a double MC, two flux ropes can be observed. Actually, for such an observed double-flux-rope structure, various explanations have been given. Marubashi (1997); Crooker et al. (1998) proposed that one flux rope (called "folded flux rope") may be observed twice because of the Parker spiral magnetic field, and show a double-flux-rope profile; this has been studied by Vandas et al. (2002) in simulation. Other authors suggested that a magnetic cloud sometimes contains two flux tubes (Fainberg et al. 1996; Osherovich et al. 1999), and then exhibits a similar double-peak structure in magnetic field strength. Apparently, how to distinguish the double MC from other types of observed double-flux-rope structures is an important problem in solarterrestrial physics since the double MC is formed because of the overtaking of two CMEs whereas the others are formed by a single CME. The differences between them will be discussed in Sect. 3 based on the results of double-MC simulation. The next section is devoted into introducing the method of simulation. Finally, we summarize the results in Sect. 4.

\section{Method and initial state}

We use the 2.5-D MHD code provided by Zheng et al. (2001a) to study the formation and propagation of a Multi-MC. The basic equations refer to the work of Jeffrey \& Taniuti (1964), and are written in a spherical coordinate system. Their dimensionless forms are given by

$$
\frac{\partial u}{\partial t}+A_{r} \frac{\partial u}{\partial r}+A_{\theta} \frac{1}{r} \frac{\partial u}{\partial \theta}+A_{\varphi} \frac{1}{r \sin \theta} \frac{\partial u}{\partial \varphi}+F=0
$$

$$
u=\left(\begin{array}{c}
\rho \\
v_{r} \\
v_{\theta} \\
v_{\varphi} \\
B_{r} \\
B_{\theta} \\
B_{\varphi} \\
p
\end{array}\right)
$$

$$
\begin{aligned}
& A_{r}=\left(\begin{array}{cccccccc}
v_{r} & \rho & 0 & 0 & 0 & 0 & 0 & 0 \\
0 & v_{r} & 0 & 0 & 0 & B_{\theta} / \rho & B_{\varphi} / \rho & \beta / 2 \rho \\
0 & 0 & v_{r} & 0 & 0 & -B_{r} / \rho & 0 & 0 \\
0 & 0 & 0 & v_{r} & 0 & 0 & -B_{r} / \rho & 0 \\
0 & 0 & 0 & 0 & v_{r} & 0 & 0 & 0 \\
0 & B_{\theta} & -B_{r} & 0 & 0 & v_{r} & 0 & 0 \\
0 & B_{\varphi} & 0 & -B_{r} & 0 & 0 & v_{r} & 0 \\
0 & \gamma p & 0 & 0 & 0 & 0 & 0 & v_{r}
\end{array}\right) \\
& A_{\theta}=\left(\begin{array}{cccccccc}
v_{\theta} & 0 & \rho & 0 & 0 & 0 & 0 & 0 \\
0 & v_{\theta} & 0 & 0 & -B_{\theta} / \rho & 0 & 0 & 0 \\
0 & 0 & v_{\theta} & 0 & B_{r} / \rho & 0 & B_{\varphi} / \rho & \beta / 2 \rho \\
0 & 0 & 0 & v_{\theta} & 0 & 0 & -B_{\theta} / \rho & 0 \\
0 & -B_{\theta} & B_{r} & 0 & v_{\theta} & 0 & 0 & 0 \\
0 & 0 & 0 & 0 & 0 & v_{\theta} & 0 & 0 \\
0 & 0 & B_{\varphi} & -B_{\theta} & 0 & 0 & v_{\theta} & 0 \\
0 & 0 & \gamma p & 0 & 0 & 0 & 0 & v_{\theta}
\end{array}\right) \\
& A_{\varphi}=\left(\begin{array}{cccccccc}
v_{\varphi} & 0 & 0 & \rho & 0 & 0 & 0 & 0 \\
0 & v_{\varphi} & 0 & 0 & -B_{\varphi} / \rho & 0 & 0 & 0 \\
0 & 0 & v_{\varphi} & 0 & 0 & B_{\varphi} / \rho & 0 & 0 \\
0 & 0 & 0 & v_{\varphi} & B_{r} / \rho & B_{\theta} / \rho & 0 & \beta / 2 \rho \\
0 & -B_{\varphi} & 0 & B_{r} & v_{\varphi} & 0 & 0 & 0 \\
0 & 0 & -B_{\varphi} & B_{\theta} & 0 & v_{\varphi} & 0 & 0 \\
0 & 0 & 0 & 0 & 0 & 0 & v_{\varphi} & 0 \\
0 & 0 & 0 & \gamma p & 0 & 0 & 0 & v_{\varphi}
\end{array}\right) \\
& F=\frac{1}{r}\left(\begin{array}{l}
\rho\left(2 v_{r}+v_{\theta} \cot \theta\right) \\
-\left(v_{\theta}^{2}+v_{\varphi}^{2}\right)+\left(B_{\theta}^{2}+B_{\varphi}^{2}\right) / \rho+\alpha / r \\
v_{r} v_{\theta}-v_{\varphi}^{2} \cot \theta-\left(B_{r} B_{\theta}-B_{\varphi}^{2} \cot \theta\right) / \rho \\
v_{\varphi}\left(v_{r}+v_{\theta} \cot \theta\right)-B_{\varphi}\left(B_{r}+B_{\theta} \cot \theta\right) / \rho \\
B_{r}\left(2 v_{r}+v_{\theta} \cot \theta\right) \\
v_{r} B_{\theta}+v_{\theta}\left(B_{r}+B_{\theta} \cot \theta\right) \\
v_{r} B_{\varphi}+v_{\varphi}\left(B_{r}+B_{\theta} \cot \theta\right) \\
\gamma p\left(2 v_{r}+v_{\theta} \cot \theta\right)
\end{array}\right),
\end{aligned}
$$

in which $t, r, \rho, v, B, p$ are normalized by $\tau_{\mathrm{A}}, R_{\mathrm{S}}, \rho_{0}, V_{\mathrm{A}}, B_{0}, p_{0}$ respectively, $\beta=8 \pi p_{0} / B_{0}^{2}, V_{\mathrm{A}}=B_{0} / \sqrt{4 \pi \rho_{0}}, \alpha=G M_{\mathrm{S}} / R_{\mathrm{S}} V_{\mathrm{A}}^{2}$, $\tau_{\mathrm{A}}=R_{\mathrm{S}} / V_{\mathrm{A}}, R_{\mathrm{S}}$ is the solar radius, $M_{\mathrm{S}}$ is the solar mass, and $G$ is the gravitational constant.

The code adopts an approximation correction splitting numerical scheme (Yanenko 1971) to model one-fluid timedependent MHD equations with the addition of artificial viscosity. More details of the code and its applications can be found in the papers by Wu et al. (2001); Zheng et al. (2001b) and $\mathrm{Li}$ et al. $(2002 \mathrm{~b}, \mathrm{a})$.

Since the axes of most magnetic clouds lie approximately in the ecliptic plane and are perpendicular to the Sun-Earth line (Lepping et al. 1990), the simulation is carried out in the heliospheric meridional plane with the assumption that the magnetic cloud is a toroid around the Sun. The computational domain is limited to the supermagnetoacoustic flow region: $20 R_{\mathrm{s}} \leq r \leq 320 R_{\mathrm{s}},-1.5^{\circ} \leq \theta \leq 181.5^{\circ}$. A $201 \times 122 \mathrm{mesh}$ is adopted with $\Delta r$ that is $0.5 R_{\mathrm{S}}$ at the base $\left(r=20 R_{\mathrm{S}}\right)$ and increases in steps of a geometrical series of common ratio 1.01 and $\Delta \theta=1.5^{\circ}$. The resolution of this mesh is high enough to distinguish the features of a typical magnetic cloud at 1 AU. The initial state is uniquely determined by the boundary conditions at the base ( $\left.r=20 R_{\mathrm{s}}\right)$ as follows: magnetic field strength 

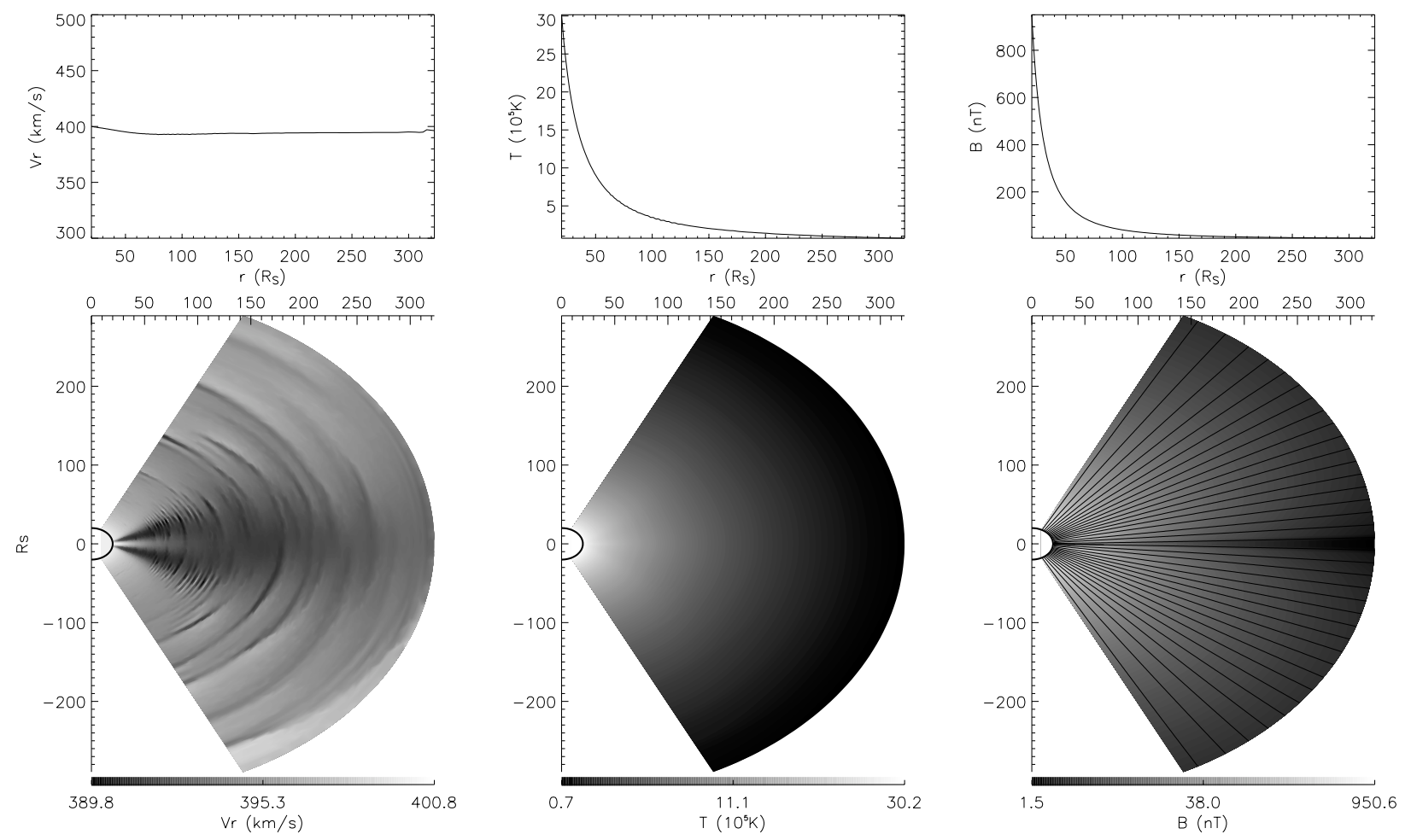

Fig. 1. Initial state of the simulation. From left to right, radial solar wind speed $V_{\mathrm{r}}$, temperature $T$ and magnetic field strength $B$ are plotted. The lower panels show the contours of these parameters, and the upper panels exhibit the profiles in the degree of $25^{\circ}$ away from the equatorial plane.

$B_{0}=1000 \mathrm{nT}$, solar wind speed $v_{0}=v_{r 0}=400 \mathrm{~km} \mathrm{~s}^{-1}$, number density $n_{0}=400 \mathrm{~cm}^{-3}$ and pressure $p_{0}=3.5 \mathrm{nPa}$. A heliospheric current sheet (HCS) is introduced by simply reversing the magnetic field direction in the region of $\theta \leq 90^{\circ}$ at the base. Because of the limitation of the code the half thickness of the HCS is $\sim 3.75^{\circ}$. The magnetic field strength gradually changes from zero to $B_{0}$ in terms of $\tanh \left(0.31\left|\theta-90^{\circ}\right|\right)$ in the HCS to keep the calculation stable. Such a thick HCS is not consistent with the real case. However, since the transverse size of the magnetic cloud is much larger than its thickness, we believe that the simulated results do not distort the primary characteristics of the formation and propagation of a Multi-MC in the interplanetary medium. Solving the 2.5-D MHD equations by the fractional step scheme with the given boundary conditions, we obtain the initial state in the computational domain, as shown in Fig. 1. From the Sun to the heliosphere the magnetic field strength and temperature decrease rapidly; the solar wind speed roughly stays at about $400 \mathrm{~km} \mathrm{~s}^{-1}$.

A cylindrical flux rope treated as a magnetic cloud is introduced at the base in the initial state. The cloud has a radius $R_{0}=3.5 R_{\mathrm{s}}$. In the inner region, $R \leq R_{0}$, the magnetic field configuration of the cloud is given by the Lundquist (1950) solution in local cylindrical coordinates $(R, \Phi, Z)$

$\left\{\begin{array}{l}B_{R}=0 \\ B_{\Phi}=3 B_{0} J_{1}(\alpha R) \\ B_{Z}=3 B_{0} J_{0}(\alpha R),\end{array}\right.$

where $\alpha=2.4 / R_{0}$, the density is 3 times the background value with the thermal pressure unchanged, and the velocity along the direction $r$ is $V_{\mathrm{c}}$. In the transition region, $R_{0}<R<2 R_{0}$, the magnetic field and the velocity are described by the solution of incompressible flow surrounding a cylinder as applied by Detman et al. (1991) and Vandas et al. (1995). Outside $2 R_{0}$, the background field and solar wind are not influenced by the cloud. To ensure that the divergence of $B$ is free, $B_{r}$ is modified when the cloud is injected into the computational domain (Detman et al. 1991). In the simplest case of a double MC, two identical magnetic clouds with different speeds propagate along the direction of $\theta=90^{\circ}$ following each other. The first cloud with a speed of $V_{\mathrm{c} 1}=v_{0}=400 \mathrm{~km} \mathrm{~s}^{-1}$ is injected at $T=0$ and the second with a speed of $V_{\mathrm{c} 2}=600 \mathrm{~km} \mathrm{~s}^{-1}$ is injected at $T=12 \mathrm{~h}$. This is consistent with the observations that the two CMEs in the March 31, 2001 event and the April 11-13, 2001 event were both all separated by half a day (Wang et al. 2003). For comparison, two isolated clouds with speeds of $400 \mathrm{~km} \mathrm{~s}^{-1}$ and $600 \mathrm{~km} \mathrm{~s}^{-1}$ respectively are also examined. Our simulation has the drawback that it cannot show the shock structure that should appear ahead of the fast cloud. This is because the code is dissipative so that it can be run in a stable manner. Hence the shock structure is numerically dissipated during computation. Since our primary aim is to study some basic features of the formation and propagation of a Multi-MC, this shortcoming will not distort the results significantly.

\section{Results and discussion}

Since the propagation of isolated clouds has been studied thoroughly, it will not be discussed here. We will emphasize on the formation and propagation of a double-MC. Figure 2 shows 


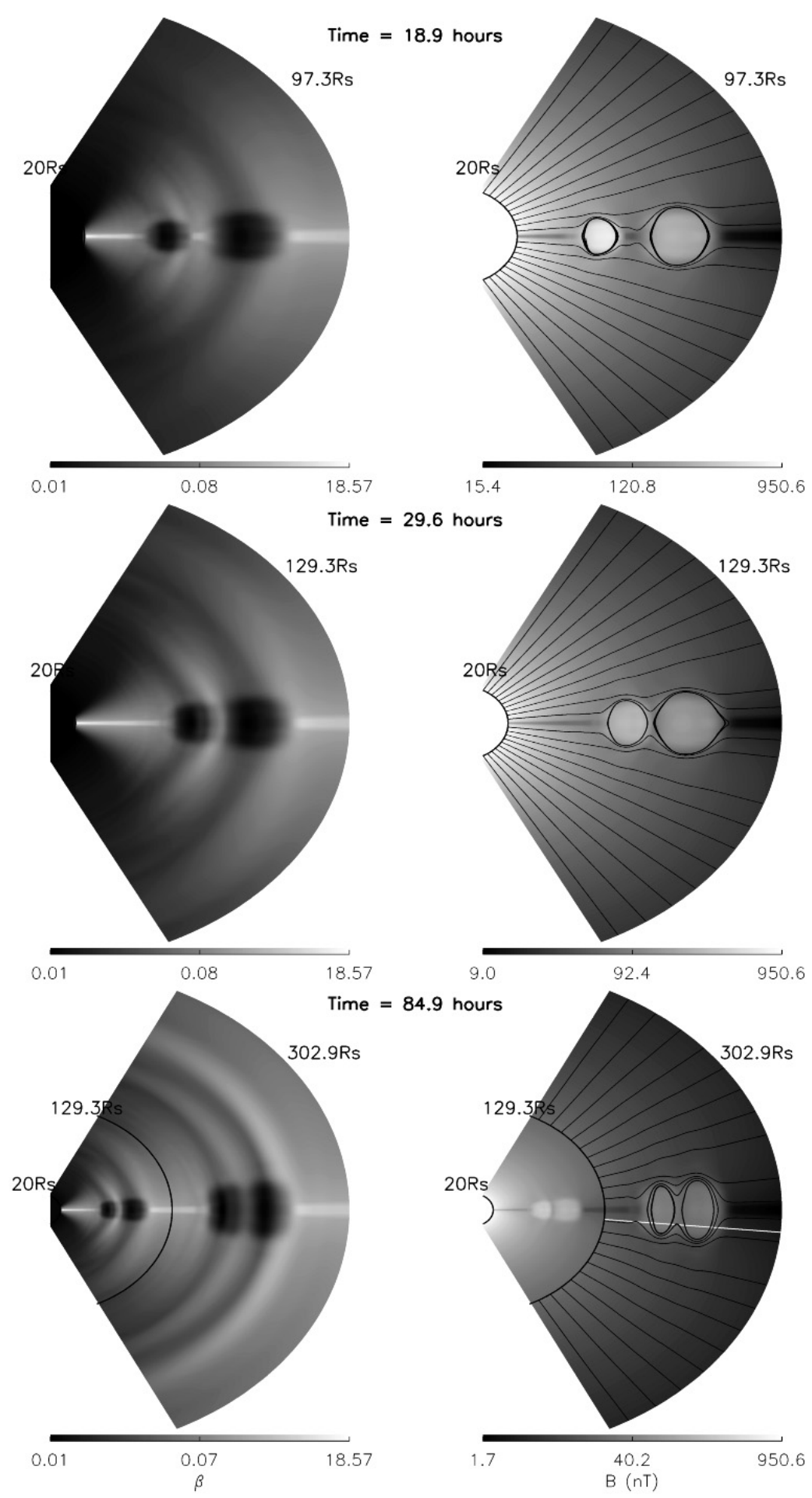

Fig. 2. The evolution of a double MC. The proton $\beta$ (left column) and total magnetic field strength (right column) with field line overlaid are given as gray-scale plots. The upper panel shows the state before the fast cloud catches up with the preceding slow cloud in the region from $20 R_{\mathrm{s}}$ to $97 R_{\mathrm{s}}$. The middle panel shows the state at $T=29.6 \mathrm{~h}$ when the fast cloud just catches up with the slow one at $\sim 78 R_{\mathrm{s}}$. The bottom panel illustrates the state where the double MC arrives at $1 \mathrm{AU}$ long after its formation. As for comparison, the plots in the middle panel are re-plotted over the bottom panel. The white line in the bottom panel denotes the observed path in the direction of $\Delta=5.25^{\circ}$, where $\Delta$ is the elevation angle relative to the equatorial plane.

the process. The observations indicate that the proton temperature inside a Multi-MC is not as low as that in a typical isolated cloud, but the proton $\beta$ still remains at a low value (Wang et al. 2003), so we prefer to use $\beta$ to study the properties of a
Multi-MC as shown in the left column of Fig. 2. The right column gives the contours of the magnetic field strength overlapped by magnetic field lines. The upper panel shows the state at $T=18.9 \mathrm{~h}$ before the fast cloud catches up with the 
preceding slow cloud in the region from $20 R_{\mathrm{S}}$ to $97 R_{\mathrm{s}}$. The two clouds can be distinguished in the panel, and the size of the preceding cloud is larger than that of the following one because of the expansion. For the regions inside the two clouds, in comparison with the background values the magnetic field strength $B$ is stronger, and the proton $\beta$ is lower. For the compressed region between the two clouds the field strength is decreased, and $\beta$ is enhanced. The middle panel shows the state at $T=29.6 \mathrm{~h}$ when the fast cloud just catches up with the slow one at a distance of $\sim 78 R_{\mathrm{s}}$ from the Sun. The time is determined by the criterion that the distance between the outmost closed magnetic field lines of the two clouds first becomes less than $2 R_{\mathrm{s}}$. The fast cloud takes $\sim 18 \mathrm{~h}$ to overtake the preceding cloud. The basic features of the two clouds are the same as those presented in the upper panel except that the region between them becomes sharper. The bottom panel presents the state where the double MC arrives at $1 \mathrm{AU}$ long after its formation. Its characteristics, such as high magnetic field strength and low proton $\beta$, and the interacting region between the two clouds are still evident. These three panels illustrate that the initial features of a magnetic cloud remain in the process of formation and propagation of a Multi-MC. Notice the reconnection of the magnetic field lines between the two sub-clouds as shown in the bottom panel. Since the field lines at the tail of preceding cloud and the head of following cloud are reverse, the numerical magnetic reconnection between them becomes unavoidable because of the limitations of the code. Numerical reconnection is impractical and sometimes may distort the facts significantly. However, even though classical resistance vanishes for collisionless plasma in the interplanetary medium, effective resistivity due to inertial effects of particles, interaction between wave and particle, and so on, are still present (e.g., Speiser 1970; Coroniti \& Eviatar 1977; Lee 1982; Sonnerup 1988). In theory, the magnetic reconnection should take place even in the interplanetary medium (Wei et al. 2003). Thus, the numerical reconnection may be treated as a substitute of the actual magnetic reconnection due to the presence of effective resistivity.

Figure 3 shows the heights in $R_{\mathrm{S}}$ as a function of time curves of the centers of clouds as determined by the smallest closed magnetic field lines inside the clouds. In the upper panel, the thick curves represent the case of a double-MC, and for comparison, the thin curves represent the cases of isolated slow and fast clouds propagating in the heliosphere. The lower panel exhibits the differences of the heights $(\Delta H)$ of the slow and fast clouds respectively between the double-MC case and the isolated cloud cases. Note that the two thick curves in the upper panel do not cross; this is because the clouds are quite large and have expanded considerable, but the curves refer to their centers. In the case of the double MC (the thick curves) the fast cloud finally becomes slower than the slow one because it is blocked by the slow cloud. As mentioned above, the interaction between the two clouds, i.e., the formation of the double MC, happens after $T \approx 30 \mathrm{~h}$, and the following fast cloud begins to slow down quickly. This can be illustrated by the obvious departure of the solid curve from zero as shown in the lower panel of Fig. 3. However, the acceleration of the preceding slow cloud is not obvious until $T \approx 83 \mathrm{~h}$ (as presented by the dashed lines),
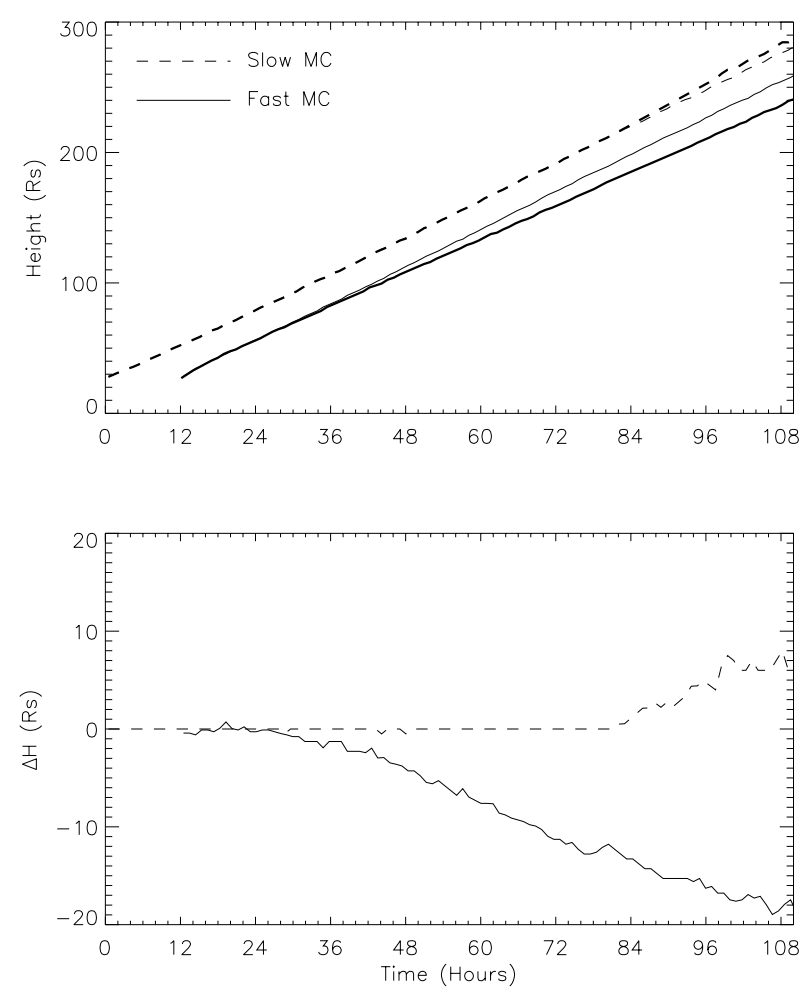

Fig. 3. The heights in $R_{\mathrm{S}}$ as a function of time curves of the centers of clouds. In the upper panel, the thick curves represent the case of double-MC, and as a comparison, the cases of isolated slow and fast clouds propagate in the heliosphere individually have been plotted as the thin curves. The lower panel exhibits the differences of the height $(\Delta H)$ of slow and fast clouds between the double-MC case and the isolated cloud cases.

which implies that the preceding cloud in the double-MC case is only accelerated slightly by the push of the following fast cloud. Here, acceleration/deceleration means that the speed becomes faster/slower than in the cases of isolated clouds. The result that the preceding slow cloud is accelerated slightly and the following fast cloud is decelerated significantly implies that the slow cloud controls the travel time of a Multi-MC structure from the Sun to $1 \mathrm{AU}$. This is consistent with the recent statistical result that $\mathrm{CME}$ interaction tends to slow the shock and the associated CME (Manoharan et al. 2004). Interaction of CMEs leads to the prediction of arrival of CMEs at $1 \mathrm{AU}$ becoming more difficult.

To compare the simulation with the in-situ observations, the changes of the interplanetary magnetic field and the solar wind parameters at $1 \mathrm{AU}$ are plotted as functions of time in Fig. 4. The measurements by a hypothetic spacecraft are along the path with the elevation angle $\Delta=5.25^{\circ}$ relative to the equatorial plane as denoted by the white line in the bottom panel of Fig. 2. The double MC arrives at 1 AU after approximately 3 days (as denoted by the two vertical lines in Fig 4). Except for the profile of the total magnetic field strength, the basic features of the double MC obtained by the simulation are the same as those derived from the theoretical model (Wang et al. 2002) as shown in Fig. 5, and are consistent with the Multi-MC (Fig. 6) observed on March 31, 2001 (Wang et al. 2003). The magnetic field strength is enhanced, and the rotation 


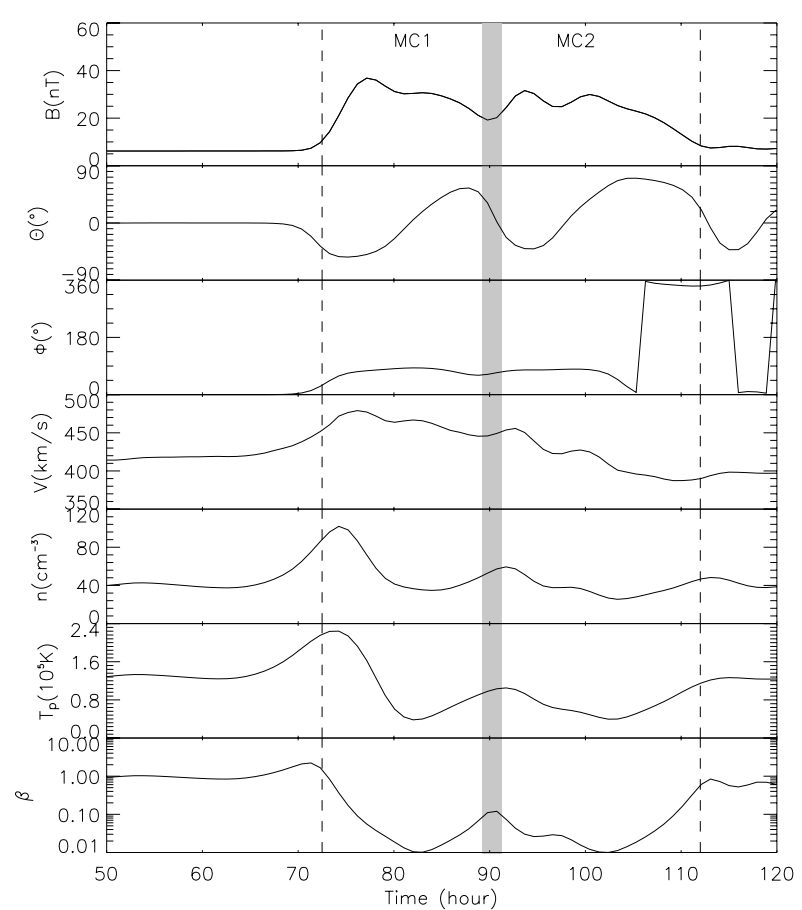

Fig. 4. In-situ measurements along a direction of $\Delta=5.25^{\circ}$ by a hypothetic spacecraft at $1 \mathrm{AU}$. From top to bottom are plotted magnetic field strength $(B)$, the elevation $(\Theta)$ and azimuthal $(\Phi)$ angles of the field direction, solar wind speed $(V)$, proton density $(n)$, proton temperature $\left(T_{\mathrm{p}}\right)$, and the ratio of proton thermal pressure to magnetic pressure $(\beta)$. The shadowed region indicates the region of interaction between the two clouds.

of the field vector is evident. The solar wind speed decreases continuously. If one applies a linear fit, one finds that the radial expansion speed of the double-cloud is about $45 \mathrm{~km} \mathrm{~s}^{-1}$, which is approximately half the local Alfvén speed and consistent with the observations (Klein \& Burlaga 1982). The proton temperature and $\beta$ within the sub-clouds are both lower than those in ambient solar wind. Between the two sub-clouds marked by a shadowed region there is the interacting region associated with a decreased magnetic field and enhanced proton temperature and $\beta$. Moreover, as in the observations, the size of the following cloud is obviously larger than that of the preceding one.

However, there is an inconsistency between the simulation and the observations of the March 31 event; a double-peak structure appears in each sub-cloud in our simulation. Inside each sub-cloud, the peaks appear at the front and the rear, and the magnetic field strength is relatively weak near the center. Figure 7 exhibits the double-peak structure more clearly. The arrows in Fig. 7 indicate the gradients of total pressure, which is dominated by magnetic pressure. The pressure peaks appear at the sites where these arrows converge. The gradients perpendicular to the magnetic field near the joint of the two subclouds indicate the compression between them. Because of the interactions among the two clouds and the ambient solar wind, the pressure inside and surrounding the clouds is not uniform. Hence, the centers of the clouds are not at the place where the total pressure is the largest, i.e., the magnetic field strength is the strongest. The double-peak structure of the cloud also

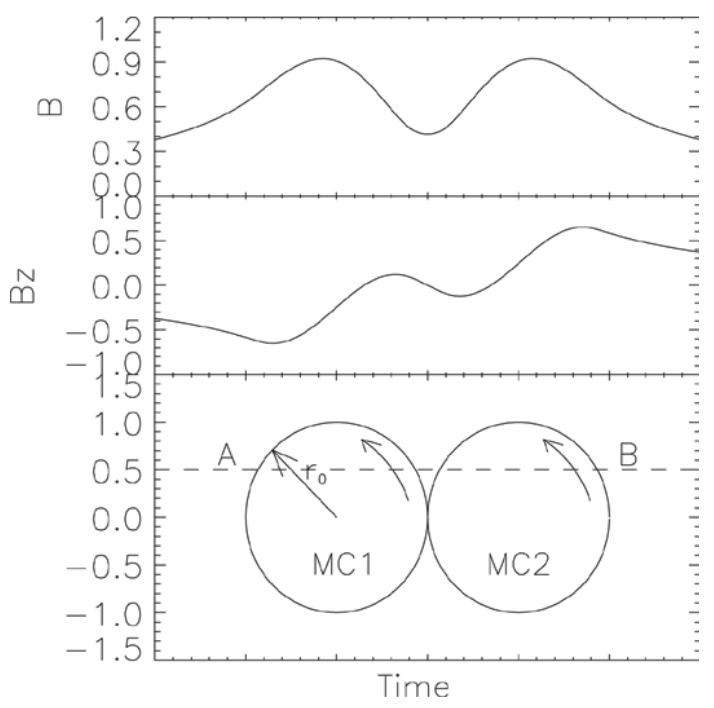

Fig. 5. The model of a double MC containing two identical clouds (Wang et al. 2002). The horizontal dashed line indicates the observed path of a hypothetic spacecraft.

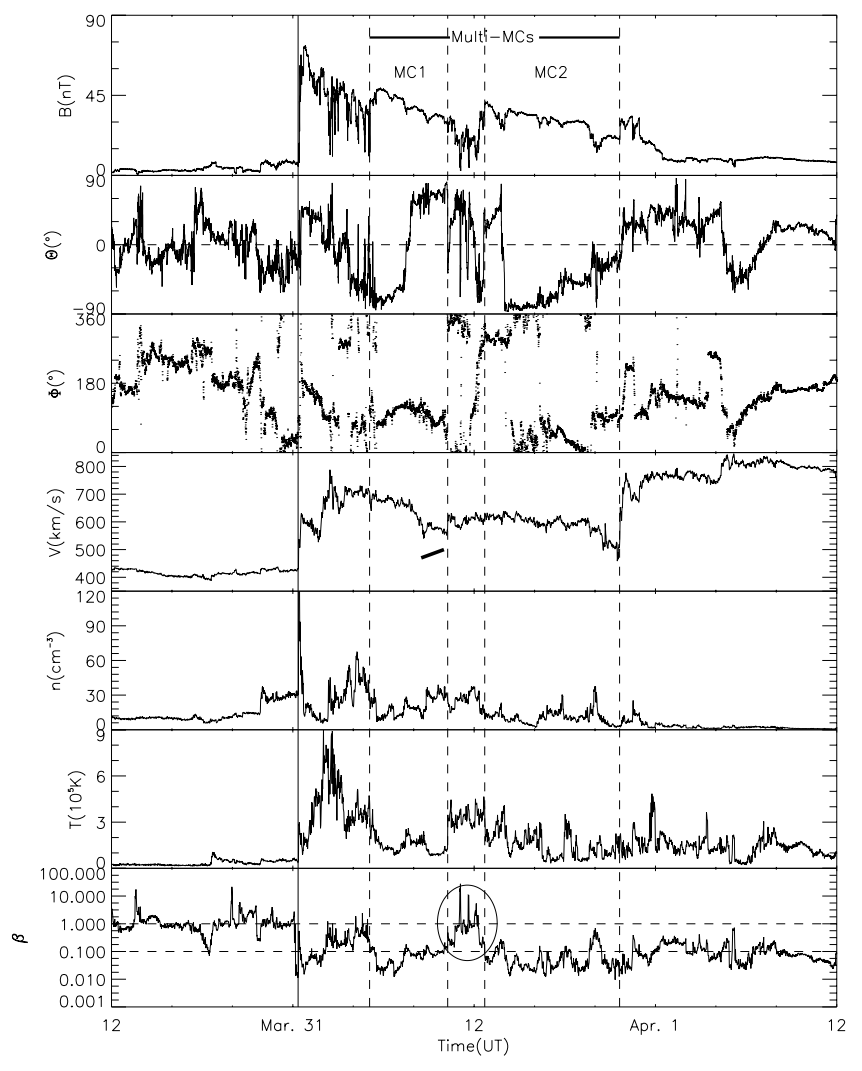

Fig. 6. The observations by the ACE spacecraft from 1200 UT 30 March to 1200 UT April 1 in GSM (cf. Wang et al. 2003) for comparison with the simulation results.

appeared in previous simulations (Vandas et al. 2002) and even in observations, for example, the Sep. 25, 1982 event and the Nov. 22, 1982 event (Lepping et al. 1990).

As mentioned in the Introduction, there are other viewpoints concerning the in-situ observed double-flux-rope structure. For the folded flux rope model proposed by Marubashi (1997); Crooker et al. (1998), such a structure is due to the 


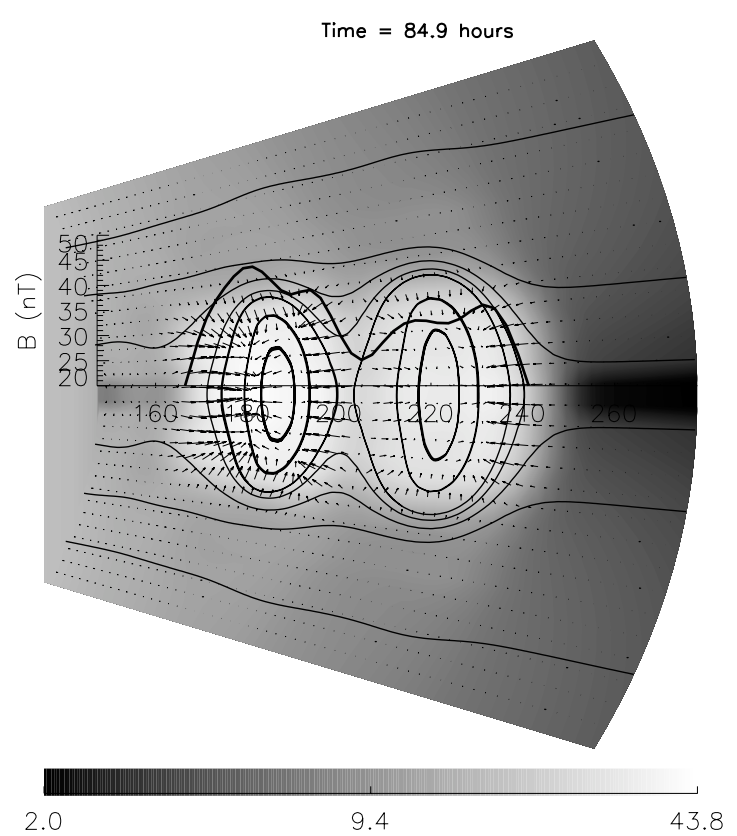

Fig. 7. The configuration of a double MC. The magnetic field strength is given in grayscale, and the value along the $x$-axis is indicated by the thick curve. The arrows denote the gradients of total pressure, which is dominated by magnetic pressure.

fact that one flux rope is observed twice. Figures 8, 10 and 11 in Vandas et al. (2002) illustrated the phenomena well. Conceptually, there are some fundamental differences between a double MC and such a folded rope structure. First, the axes of two observed flux ropes in a folded rope structure should be roughly in the ecliptic plane, whereas the two cloud axes in a double-MC may lie in arbitrary planes. Second, the orientation of the elevation angle of the magnetic field vector within the folded rope structure must be south-north-north-south or northsouth-south-north, whereas the double MC is not restricted by this rule. Third, the signs of the helicity of the two ropes in the folded rope structure must be the same, whereas that is not necessary for a double MC. The first difference follows from the spiral background magnetic field, which causes the looplike cloud to be deformed and therefore probably is observed twice. The other two differences are inferred from the basic fact that the folded rope structure is formed by only one CME but a double MC is composed of two CMEs. In addition to the above differences, some others can be found by comparing Fig. 10 in the paper of Vandas et al. (2002) with Fig. 4 and 6 in the present paper. There is no compression and no evident interacting region between the two observed flux ropes in the folded-rope structure, and sometimes the two ropes can be a long distance away from each other. As for the scenario in which a magnetic cloud contains two flux tubes, it is more similar to a double-MC in in-situ observations. Fortunately, there is a fundamental difference between them, i.e., a double MC is associated with two solar events but the other scenario is not. Based on the solar observations, two associated CMEs can be found in all cases of the double MC in Wang et al. (2003). If only one CME is responsible for the two observed flux ropes, it is difficult to explain where the second front-side halo CME goes. Here, the likelihood of a double MC in the interplanetary medium is also supported by the result of the simulation that two successive clouds initially separated by 12 $\mathrm{h}$ do form a double $\mathrm{MC}$ at $1 \mathrm{AU}$, which is consistent with the observations. Furthermore, triple and multiple-magneticcloud structures may certainly be expected in the interplanetary medium too.

In addition, the observations from SOHO reveal that two CMEs initially apart by several hours will cannibalize each other in the inner corona (Gopalswamy et al. 2001). Simulation of the interaction of two CMEs has been performed by $\mathrm{Wu}$ et al. (2002) to study this phenomenon. The simulation results suggest that such cannibalization is caused by magnetic reconnection. This process is similar to double-MC formation, but the former occurs near the Sun and the two CMEs will merge quickly, whereas the latter occurs in the interplanetary medium and the process of merging becomes very slow. Since the characteristic scale and the density in the interplanetary medium are much larger and lower, respectively, than near the Sun, the frozen condition is satisfied better in the interplanetary medium, and the magnetic reconnection rate should therefore be far smaller than in the inner corona. Thus, the phenomena of CME cannibalization near the Sun and Multi-MCs in the interplanetary medium are both reasonable. Moreover, one can find that the two cannibalized CMEs were initially separated by only a few hours (Gopalswamy et al. 2001; Wu et al. 2002), whereas the two CMEs forming the double MC in Wang et al. (2003) were initially separated by more than ten hours. Is a long interval between successive CMEs the condition for forming Multi-MC in the interplanetary space? Will the sub-clouds within a Multi-MC eventually merge? Wang et al. (2004b) proposed that there are at least two conditions for two successive CMEs to form a Multi-MC in the interplanetary medium: (1) the second CME should be faster than the first, and (2) they should be initially separated by a moderate interval at the beginning. These conditions need to be further testified by more observations and simulations.

\section{Conclusions and summary}

A 2.5-D MHD simulation has been presented in this paper to study the formation and propagation of double MC. The two successive magnetic clouds separated by $12 \mathrm{~h}$ at the beginning touch each other $18 \mathrm{~h}$ later and arrive at $1 \mathrm{AU}$ after approximately 3 days. Compared to the cases of isolated clouds, the acceleration of the preceding slow cloud due to the push of the following fast cloud is not obvious, whereas the slowdown of the following fast cloud is very significant. This result implies that the travel time of a Multi-MC structure is controlled by the preceding slow MC. Moreover, the simulation demonstrates most of the primary observational characteristics of Multi-MC. These results confirm the existence of Multi-MC in the interplanetary medium. We find that the differences between Multi-MC and other types of in-situ observed doubleflux-rope structure are definite and observable. In addition, a comparison between Multi-MC and CME cannibalization near the Sun has been made. These two phenomena are both likely. These results and discussions advance our understanding of the solar-terrestrial physical processes. 
Acknowledgements. We acknowledge the use of the data from ACE spacecraft. We also express our thanks to the referee for his constructive suggestions. This work is supported by the Chinese Academy of Sciences (KZCX2-SW-136), the National Natural Science Foundation of China (40404014, 40336052, 40336053 and 40274050), and the State Ministry of Science and Technology of China (G2000078405).

\section{References}

Burlaga, L., Sittler, E., Mariani, F., \& Schwenn, R. 1981, J. Geophys. Res., 86(A8), 6673

Burlaga, L. F. 1988, J. Geophys. Res., 93, 7217

Burlaga, L. F., Behannon, K. W., \& Klein, L. W. 1987, J. Geophys. Res., 92, 5725

Burlaga, L. F., Skoug, R. M., Smith, C. W., et al. 2001, J. Geophys. Res., 106, 20957

Coroniti, F. V., \& Eviatar, A. 1977, ApJS, 33, 189

Crooker, N. V., Gosling, J. T., \& Kahler, S. W. 1998, J. Geophys. Res., 103,301

Detman, T. R., Dryer, M., Yeh, T., et al. 1991, J. Geophys. Res., 96, 9531

Fainberg, J., Osherovich, V. A., Stone, R. G., MacDowall, R. J., \& Balogh, A. 1996, in Sol. Wind Eight, ed. D. Winterhalter, J. T. Gosling, S. R. Habbal, W. S. Kurth, \& M. Neugebauer, Am. Inst. of Phys. (NY: Woodbury), 554

Farrugia, C. J., Richardson, I. G., Burlaga, L. F., Lepping, R. P., \& Osherovich, V. A. 1993, J. Geophys. Res., 98, 15497

Goldstein, H. 1983, in Sol. Wind Five, NASA Conf. Publ., 2280, Washington DC, 731

Gopalswamy, N. 2003, Geophys. Res. Lett., 30(12), 8013

Gopalswamy, N., Yashiro, S., Kaiser, M. L., Howard, R. A., \& Bougeret, J. L. 2001, ApJ, 548, L91

Gopalswamy, N., Yashiro, S., Kaiser, M. L., Howard, R. A., \& Bougeret, J.-L. 2002a, Geophys. Res. Lett., 29(8), 1265

Gopalswamy, N., Yashiro, S., Michalek, G., et al. 2002b, ApJ, 572, L103

Jeffrey, A., \& Taniuti, T. 1964, Non-linear wave propagation with applications to physics and magnetohydrodynamics (New York, London: Academic Press)

Klein, L. W., \& Burlaga, L. F. 1982, J. Geophys. Res., 87, 613

Kumar, A., \& Rust, D. M. 1996, J. Geophys. Res., 101, 15667

Larson, D. E., Lin, R. P., McTiernan, J. M., et al. 1997, Geophys. Res. Lett., 24(15), 1911

Lee, L. C. 1982, Geophys. Res. Lett., 9, 1159

Lepping, R. P., Jones, J. A., \& Burlaga, L. F. 1990, J. Geophys. Res., 95,11957
Li, B., Zheng, H. N., \& Wang, S. 2002a, Chinese Astron. Astrophys., 26,458

Li, B., Zheng, H. N., \& Wang, S. 2002b, Chinese Phys. Lett., 19, 1639

Lundquist, S. 1950, Ark. Fys., 2, 361

Manoharan, P. K., Gopalswamy, N., Yashiro, S., et al. 2004, J. Geophys. Res., 109, A06109

Marubashi, K. 1997, in Coronal Mass Ejections, ed. N. Crooker, J. A. Joselyn, \& J. Feynman, Geophys. Monogr. Ser., 99, AGU, 147

Osherovich, V. A., \& Burlaga, L. F. 1997, in Coronal mass ejections, ed. N. Crooker, J. A. Joselyn, \& J. Feynman, Geophys. Monogr. Ser., 99, AGU, 157

Osherovich, V. A., Fainberg, J., \& Stone, R. G. 1999, Geophys. Res. Lett., 26(3), 401

Sonnerup, B. U. Ö. 1988, Comput. Phys. Comm., 49, 143

Speiser, T. W. 1970, Planet. Space Sci., 18, 613

Vandas, M., Fischer, S., Dryer, M., Smith, Z., \& Detman, T. 1995, J. Geophys. Res., 100, 12285

Vandas, M., Fischer, S., Dryer, M., Smith, Z., \& Detman, T. 1996, J. Geophys. Res., 101, 2505

Vandas, M., Fischer, S., Odstrcil, D., et al. 1997, in Coronal mass ejections, ed. N. Crooker, J. A. Joselyn, \& J. Feynman, Geophys. Monogr. Ser., 99, AGU, 169

Vandas, M., Odstrcil, D., \& Watari, S. 2002, J. Geophys. Res., 107(A9), 1236

Wang, Y., Ye, P., \& Wang, S. 2004a, Chinese J. Geophys., 47, 417

Wang, Y., Ye, P., Wang, S., \& Zheng, H. 2004b, in The 35th COSPAR Scientific Assembly, COSPAR, Paris, France

Wang, Y. M., Wang, S., \& Ye, P. Z. 2002, Sol. Phys., 211, 333

Wang, Y. M., Ye, P. Z., \& Wang, S. 2003, J. Geophys. Res., 108(A10), 1370

Wei, F., Hu, Q., Feng, X., \& Fan, Q. 2003, Space Sci. Rev., 107, 107

Wu, S. T., Wang, A. H., \& Gopalswamy, N. 2002, in Magnetic Coupling of the Solar Atmosphere, Euroconference \& IAU Coll., 188, ESA SP-505, Santorini, Greece, 227

Wu, S. T., Zheng, H. N., Wang, S., et al. 2001, J. Geophys. Res., 106, 25089

Yanenko, N. N. 1971, The method of fractional steps, the solution of problems of mathematical physics in several variables (New York, HeideBerg, Berlin: Springer-Verlag), 149

Zheng, H., Wu, S. T., \& Wang, S. 2001a, preprint

Zheng, H. N., Wang, S., Wu, S. T., \& Li, B. 2001b, Chinese Phys. Lett., 18, 1624 